\title{
Clinical presentation and magnetic resonance imaging characteristics of lymphocytic hypophysitis: a systematic review with meta-analysis
}

\author{
Jia-Sheng Ju' ${ }^{1}$ Tao Cui ${ }^{2}$, Jing Zhao ${ }^{2}$, Jian-Liang Chen ${ }^{1}$, Hai-Bing Ju
}

${ }^{1}$ Department of Neurosurgery, The Eighth Affiliated Hospital of Sun Yet-sen University, Shenzhen, China

2Department of Endocrinology, $920^{\text {th }}$ Hospital of Joint Logistics Support Force of the Chinese People's Liberation Army, Kunming, China

Submitted: 20 April 2021; Accepted: 7 December 2021

Online publication: 14 December 2021

Arch Med Sci

DOI: https://doi.org/10.5114/aoms/144628

Copyright $\odot 2022$ Termedia \& Banach

\section{Abstract}

Introduction: This meta-analysis was performed to analyze the clinical presentation, magnetic resonance imaging (MRI) characteristics, and the management of lymphocytic hypophysitis (LYH).

Material and methods: Two researchers searched the Cochrane Controlled Trials Register (CCTR), Ovid, PubMed, Excerpta Medica Database (EMBASE), and the Chinese Biomedical Literature Databases (CBM). The databases were searched from January 2010 to December 2020. References and related reviews found in the literature were also retrieved.

Results: The meta-analysis showed that the percentage of women in LYH patients was $78 \%$. Lymphocytic hypophysitis was associated with pregnancy in $15 \%$ of the female patients, with headache $(49 \%)$ and symptoms of central diabetes insipidus (CDI) (45\%) being the most frequent presentation. In $24 \%$ of the $\mathrm{LYH}$ patients, there was an association with another autoimmune disease. The incidence of secondary hypogonadism, secondary hypoadrenalism, secondary hypothyroidism, and growth hormone deficit was $54 \%, 49 \%$, $43 \%$, and $22 \%$, respectively. Pituitary contrast enhancement (63\%), symmetrical pituitary enlargement (60\%), thickening of the pituitary stalk $(58 \%)$, sella mass or suprasellar extension (58\%), and loss of posterior pituitary hyperintensity (50\%) were typical MRI findings. Regarding LYH treatment, the percentage of patients who had observation or hormone replacement, steroid therapy, and surgery was $43 \%, 36 \%$, and $34 \%$, respectively.

Conclusions: It is of great significance to fully understand the clinical characteristics of lymphocytic hypophysitis, reduce missed diagnosis and misdiagnosis, avoid unnecessary surgery and maintain normal pituitary function.

Key words: lymphocytic hypophysitis, clinical characteristics, magnetic resonance imaging, management, pituitary function.

\section{Introduction}

Lymphocytic hypophysitis (LYH) is a rare disease of presumed autoimmune etiology characterized by lymphocytic infiltration of the pituitary gland followed by fibrosis [1]. The presenting symptoms correspond to the location of the lesion. The most common presenting symptoms in female patients are amenorrhea and failure to lactate secondary to

\author{
Corresponding authors: \\ Hai-Bing Ju, MM \\ Department of Endocrinology \\ $920^{\text {th }}$ Hospital of Joint \\ Logistics Support Force \\ of the Chinese People's \\ Liberation Army \\ No. 212 Daguan Street, \\ Xishan District, \\ Kunming, 650032, China \\ Phone: +8613187865520 \\ Fax: +8613187865520 \\ E-mail: juhaibimg@163.com \\ Jian-Liang Chen, MD \\ Department of Neurosurgery \\ The Eighth Affiliated Hospital \\ of Sun Yet-sen University \\ No. 3025 Shennanzhong \\ Street, Futian District \\ Shenzhen 518000, China \\ Phone: +8613902974061 \\ Fax: +86 13902974061 \\ E-mail: chemjl@126.com
}


hypopituitarism, as the inflammatory process damages both the anterior and posterior pituitary gland, along with the pituitary stalk. There may also be neurological symptoms such as headaches and visual field defects secondary to the rapid expansion of the infiltrated pituitary gland. LYH has an estimated annual incidence of approximately 0.1 cases per million population, based on extrapolated data sourced from the UK, where $0.8 \%$ of consecutive pituitary operations were found to demonstrate LYH histologically [2]. However, its true incidence in the general population may be underestimated.

Due to its rarity, clinical data to guide the diagnosis and treatment of LYH have been limited, and there are no specific guidelines on management. Gutenberg noted that approximately $40 \%$ of patients with LYH were diagnosed as pituitary macroadenomas on the basis of radiology and underwent unnecessary surgery [3]. Many of the landmark articles on LYH to date have only included those patients undergoing surgery with a confirmed histological diagnosis. However, as $\mathrm{LYH}$ is an autoimmune disease, most patients do not need surgical treatment [4]. Therefore, it is of great practical significance to fully understand the clinical characteristics of $\mathrm{LYH}$ and to improve the diagnostic rate without the need for pathological evidence. The aim of this meta-analysis was to analyze the clinical presentation, endocrine status, magnetic resonance imaging (MRI) characteristics, and management of $\mathrm{LYH}$, in order to improve clinician awareness of LYH and the prognosis and quality of life of patients with the disease, and to reduce healthcare costs.

\section{Material and methods}

This meta-analysis was conducted using the Preferred Reporting Items for Systematic Reviews and Meta-Analyses (PRISM) guidelines. Author's statement: all supporting data are included in the article and its supplementary materials. This study did not require ethical approval because all analyses were based on previously published studies.

\section{Literature retrieval and screening, data extraction and quality evaluation}

Two researchers searched the Cochrane Controlled Trials Register (CCTR), Ovid, PubMed, Excerpta Medica Database (EMBASE), and the Chinese Biomedical Literature Databases (CBM). The databases were searched from January 2010 to December 2020. References and related reviews found in the literature were also retrieved. Primary search terms were hypophysitis, primary hypophysitis, autoimmune hypophysitis, lymphocytic hypophysitis, clinical features, clinical characteristic, clinical presentation, clinical manifestations, MRI, management, and treatment.

Two researchers independently conducted literature screening, data extraction, and quality evaluation. Results were cross-checked and any disagreements were mediated by discussion or with the assistance of a third-party researcher. All literature was screened sequentially for inclusion and exclusion criteria.

\section{Inclusion and exclusion criteria}

Inclusion criteria: All clinical studies that report the clinical characteristics of patients with $\mathrm{LYH}$. The diagnostic criteria for $\mathrm{LYH}$ are based on pathological and clinical findings. The criteria for judging the endocrine function of each pituitary target gland conform to the diagnostic criteria in currently recognized authoritative international guidelines. These studies report at least any clinical feature of $\mathrm{LYH}$, including gender, clinical manifestations, pituitary function changes, MRI manifestations, other concomitant autoimmune diseases, or treatment. The evaluation of pituitary function depends on the detection of pituitary target gland hormones and the pituitary function test as follows:

Growth hormone $(\mathrm{GH})$ : $\mathrm{GH}$ secretion function was evaluated by the insulin hypoglycemic stimulation test and clonidine test, and $\mathrm{GH}$ peak $<5 \mathrm{ng} / \mathrm{ml}$ and/or IGF-1 decrease was judged as GH deficiency.

Pituitary-thyroid axis: Free triiodothyronine (FT3), total triiodothyronine (TT3), free thyroxine (FT4), total thyroxine (TT4), and thyroid stimulating hormone (TSH) were assessed for thyroid function.

Pituitary-adrenal axis: Assessment was made by measuring 24-hour urine free cortisol (24hUFC), serum cortisol and ACTH rhythm (8 am, 4 pm, 0 $\mathrm{am})$, and performing the insulin hypoglycemic stimulation test. In the insulin hypoglycemic stimulation test, peak cortisol < $18 \mu \mathrm{g} / \mathrm{dl}$ was judged as adrenal hypoglycemic dysfunction.

Pituitary-gonadal axis: Assessment was made by measuring luteinizing hormone, follicle stimulating hormone, prolactin, estradiol, progesterone, testosterone levels, and performing the gonadotropin-releasing hormone $(\mathrm{GnRH})$ excitation test. Hypogonadotropism was determined by the $\mathrm{GnRH}$ stimulation test when luteinizing hormone $(\mathrm{LH})$ increased by less than 3-6 times or follicle stimulating hormone (FSH) increased by less than $50 \%$.

Posterior pituitary function was assessed by measuring urine specific gravity and urine osmotic pressure after the vasopressin test.

Exclusion criteria: Case reports, abstracts, letters, animal experiments, conference literature, duplicate publications, and literature with incomplete original data where it is not possible to extract data. 


\section{Statistical analysis}

Meta-analysis was performed using Stata 15 software. The combined rate of event incidence was used as the pooled effect size. All effect sizes were expressed as a $95 \%$ confidence interval (CI). A $\chi^{2}$ test was used to test the heterogeneity of all included study literature. The significance level was set as $p=0.10$. When $p>0.10$ and $P^{2}<50 \%$, the study results were not heterogenous and a fixed effects model was used for combined analysis. When $p<0.10$ and $l^{2}>50 \%$, the study results were heterogenous and a random effects model was used for combined analysis.

\section{Results}

\section{Literature retrieval results}

Preliminary inspection identified 326 study articles. After reading the title, abstract, and full text, those studies that did not meet the inclusion criteria were excluded. The final analysis group included 17 clinical studies with a total of 364 subjects with LYH [5-21]. The basic characteristics of the study included in this analysis are shown in Table I, and the baseline data are shown in Table II.

\section{Patient features}

Sixteen studies [6-21], involving a total of 355 patients, reported the ratio of males to females.
Heterogeneity analysis revealed no statistical heterogeneity among these 16 studies; thus, the fixed effects model was used for combined analysis. The results showed that the percentage of women among $\mathrm{LYH}$ patients was $78 \%(95 \% \mathrm{Cl}$ : 0.73-0.82) (Figure 1).

Thirteen studies [5-10, 15-21], involving a total of 241 patients, reported that LYH onset in female patients is related to pregnancy. Fifteen studies [5, 7-12, 14-21], involving a total of 340 patients, reported an association between $\mathrm{LYH}$ and other autoimmune diseases. In both study series, heterogeneity analysis revealed that there was statistical heterogeneity; thus, the random effects model was used for combined analysis. The results showed that in $15 \%$ of females, LYH was related to pregnancy (95\% Cl: 0.08-0.23) (Figure 2). The incidence of $\mathrm{LYH}$ patients with other autoimmune diseases was 0.24 (95\% Cl: 0.15-0.32), with Hashimoto's thyroiditis being the most common coexisting autoimmune disease.

\section{Sella mass-effect symptoms}

All 17 studies [5-21], involving a total of 364 patients, reported headache as a symptom caused by $\mathrm{LYH}$. Fifteen studies [6-13, 15-21], involving a total of 350 patients, reported visual field loss and visual impairment caused by LYH. In both study series, heterogeneity analysis revealed that

Table I. Basic information of published literature

\begin{tabular}{|c|c|c|c|c|c|}
\hline Study ID & Journal & $\begin{array}{c}\text { Publication } \\
\text { year }\end{array}$ & $\begin{array}{l}\text { Publication } \\
\text { level }\end{array}$ & $\begin{array}{l}\text { Research design } \\
\text { and methodology }\end{array}$ & $\begin{array}{c}\text { Dates when } \\
\text { cases collected }\end{array}$ \\
\hline An 2013 & $\begin{array}{c}\text { Chinese Journal of Chronic } \\
\text { Disease Prevention and Control }\end{array}$ & 2013 & Full text & Retrospective study & 2008-2011 \\
\hline Liu 2013 & $\begin{array}{c}\text { Chinese Journal of General } \\
\text { Practitioners }\end{array}$ & 2013 & Full text & Retrospective study & 2010-2011 \\
\hline Feng 2019 & $\begin{array}{l}\text { Chinese Journals of Practical } \\
\text { Medicine }\end{array}$ & 2019 & Full text & Retrospective study & 2013-2018 \\
\hline Wei 2018 & Chinese Medical Journal & 2018 & Full text & Retrospective study & 2001-2017 \\
\hline Xu 2020 & $\begin{array}{l}\text { Journal of Stereotactic and } \\
\text { Functional Neurosurgery }\end{array}$ & 2020 & Full text & Retrospective study & 2010-2019 \\
\hline Tirosh 2016 & Endocr Pract & 2016 & Full text & Retrospective study & $2008-2014$ \\
\hline Zhu 2019 & World Neurosurg & 2019 & Full text & Retrospective study & 2011-2018 \\
\hline Kyriacou 2017 & Pituitary & 2017 & Full text & $\begin{array}{l}\text { Multicenter cohort } \\
\text { study }\end{array}$ & $2008-2011$ \\
\hline Wang 2017 & Clin Endocrinol & 2017 & Full text & Retrospective study & 1999-2016 \\
\hline Iuliano 2011 & Expert Rev Endocrinol Metab & 2011 & Full text & Retrospective study & 2008-2011 \\
\hline Honegger 2015 & J Clin Endocrinol Metab & 2015 & Full text & Retrospective study & 2000-2013 \\
\hline Chiloiro 2017 & Neuroendocrinology & 2017 & Full text & Retrospective study & $2011-2015$ \\
\hline Khare 2015 & Pituitary & 2015 & Full text & Retrospective study & 2006-2012 \\
\hline Park 2014 & Endocrinol Metab & 2014 & Full text & Retrospective study & 2001-2013 \\
\hline Atkins 2020 & Endocrine Research & 2020 & Full text & Retrospective study & 2000-2017 \\
\hline Amereller 2020 & Exp Clin Endocrinol Diabetes & 2020 & Full text & Retrospective study & 1997-2016 \\
\hline Hanife 2020 & Endocrine Care & 2020 & Full text & Retrospective study & 1999-2017 \\
\hline
\end{tabular}


Table II. Baseline data of included studies

\begin{tabular}{|lcccc|}
\hline Study ID & Nationality & $\begin{array}{c}\text { Number of patients } \\
(\text { men/women })\end{array}$ & Average age [years] & Course of disease \\
\hline An 2013 & Chinese & $4 / 11$ & Median 46 & Median 6 months \\
\hline Liu 2013 & Chinese & $1 / 4$ & $19-61$ & 2-24 months \\
\hline Feng 2019 & Chinese & $7 / 25$ & Median 31 & Median 6 months \\
\hline Wei 2018 & Chinese & $5 / 13$ & Mean 36.3 & Median 3.5 months \\
\hline Xu 2020 & Chinese & $3 / 1$ & Mean 46 & Mean 2 months \\
\hline Tirosh 2016 & Israeli & $0 / 9$ & Mean 33.7 & Unclear \\
\hline Zhu 2019 & Chinese & $4 / 16$ & Median 37 & Mean 5 months \\
\hline Kyriacou 2017 & British & $3 / 19$ & Median 37.5 & Median 3 months \\
\hline Wang 2017 & Chinese & $17 / 33$ & Mean 37.2 & Median 4 months \\
\hline luliano 2011 & American & $3 / 4$ & Mean 44 & Unclear \\
\hline Honegger 2015 & German & $5 / 19$ & Mean 40.7 & Unclear \\
\hline Chiloiro 2017 & Italian & $4 / 17$ & Mean 40 & Unclear \\
\hline Khare 2015 & Indian & $3 / 21$ & Mean 48 & Unclear \\
\hline Park 2014 & Korean & $5 / 17$ & Mean 38 & Mean 12 weeks \\
\hline Atkins 2020 & Canadian & $1 / 10$ & Mean 45 & Unclear \\
\hline Amereller 2020 & German & $16 / 44$ & Median 41.5 & Median 18 months \\
\hline Hanife 2020 & Turkish & $5 / 15$ & &
\end{tabular}

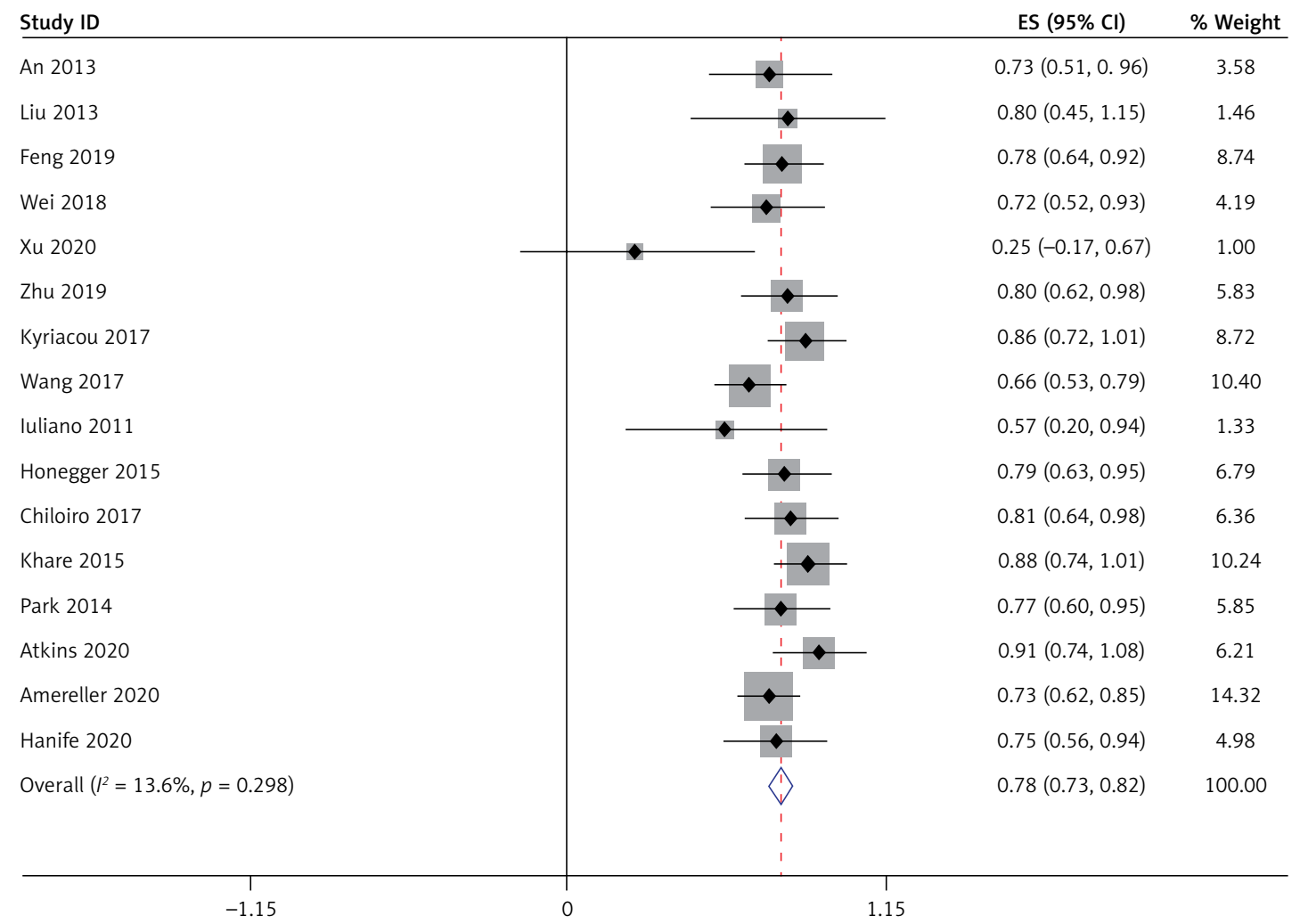

Figure 1. Female proportion

there was statistical heterogeneity; thus, the random effects model was used for combined analysis. The results showed that the incidence of headache was 0.49 (95\% Cl: 0.40-0.58) (Figure 3). The incidence of visual field loss and visual impairment was 0.26 (95\% Cl: 0.19-0.33).

\section{Evaluation of pituitary function}

Evaluation of anterior pituitary target gland function

All 17 studies [5-21], involving a total of 364 patients, reported the occurrence of hypoadrenalism, 


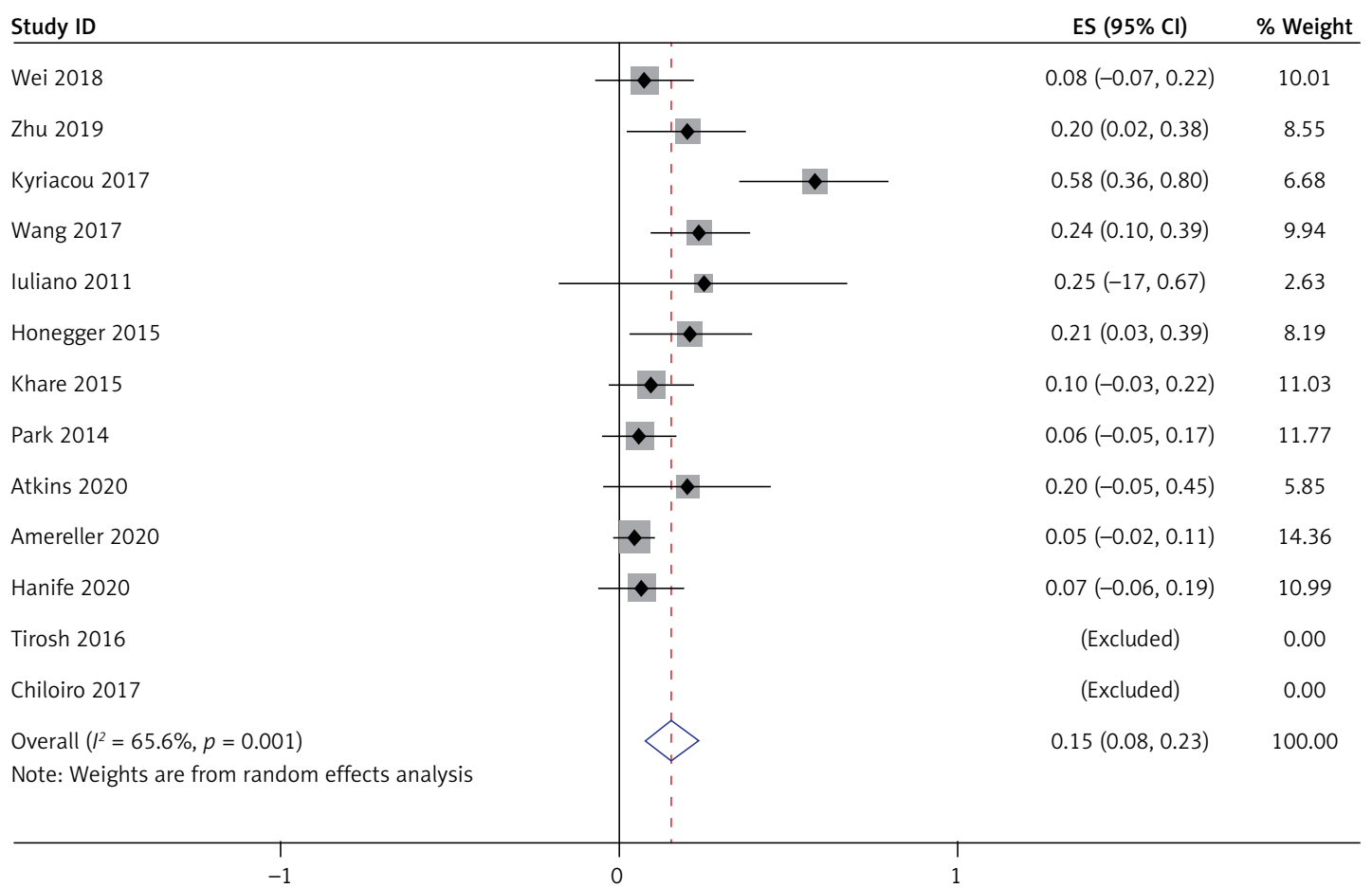

Figure 2. Proportion associated with pregnancy

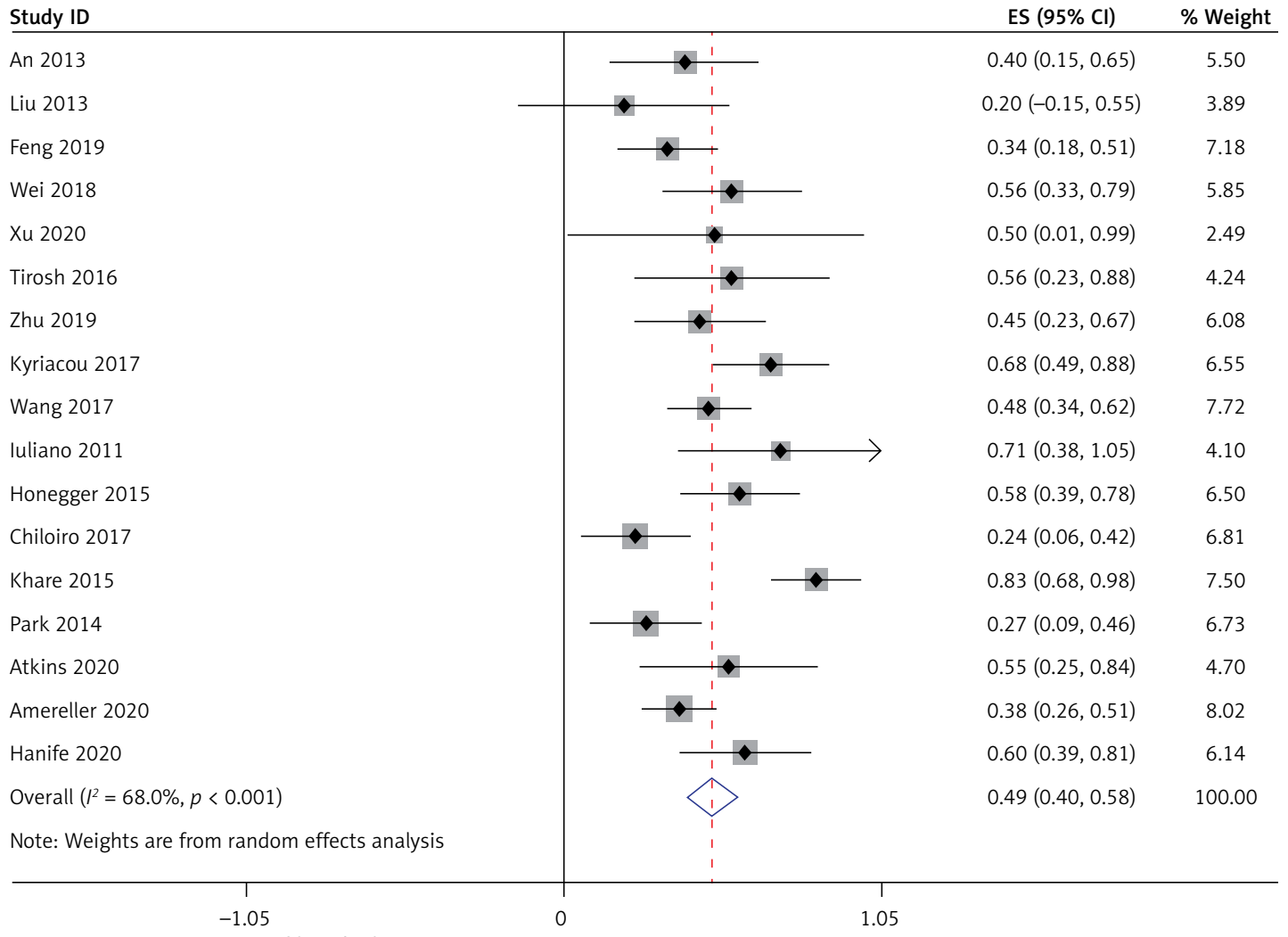

Figure 3. Proportion of headache

hypothyroidism, and hypogonadism in LYH patients. Heterogeneity analysis revealed that there was statistical heterogeneity among these 17 studies; thus, the random effects model was used for combined analysis. The results showed that the incidence of secondary hypoadrenalism in $\mathrm{LYH}$ patients was 0.49 (95\% Cl: 0.38-0.60) (Figure 4). The incidence of secondary hypothyroidism was 0.43 (95\% Cl: 0.35-0.50), and the incidence of secondary hypogonadism was 0.54 (95\% Cl: $0.42-0.65)$. 


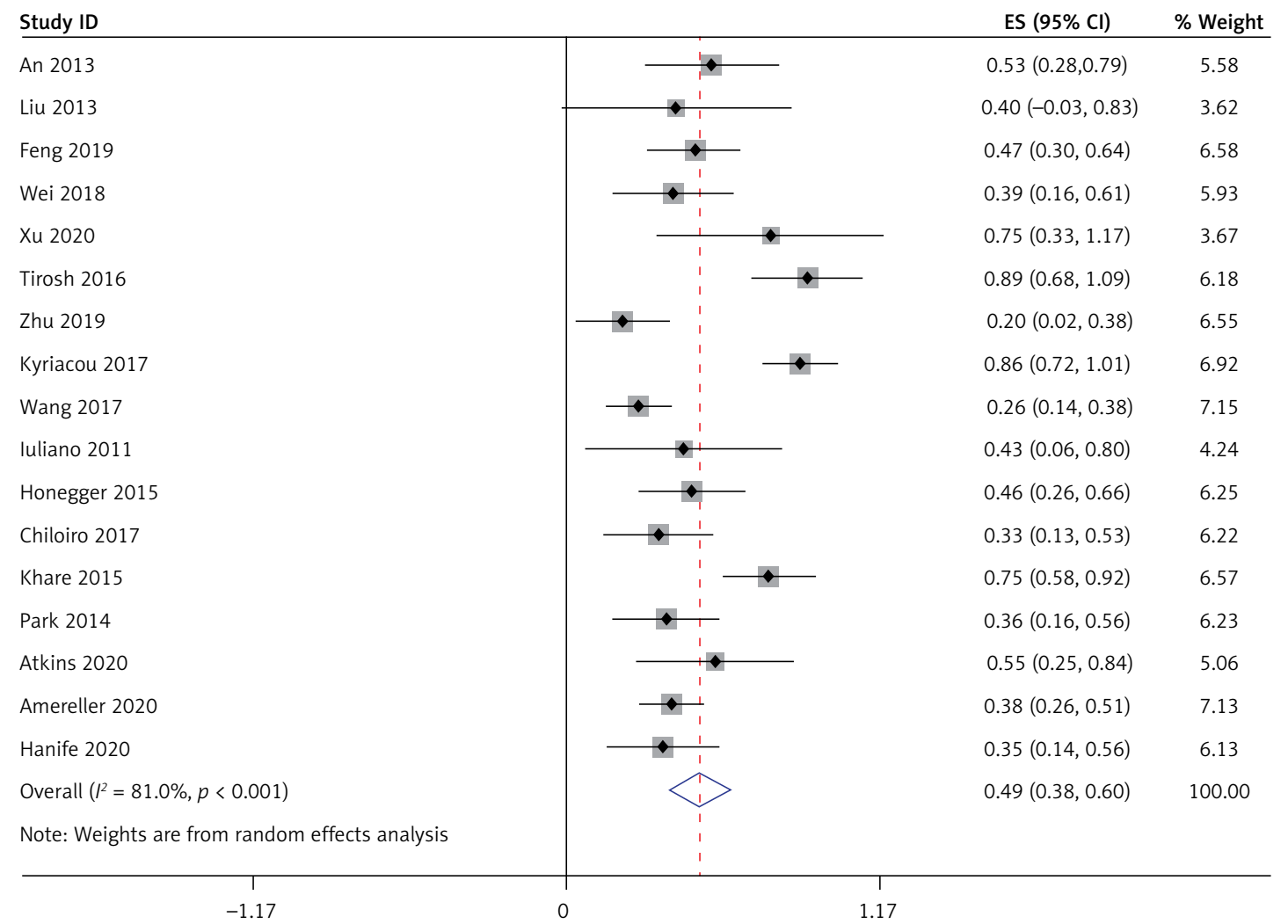

Figure 4 . Incidence of secondary hypoadrenalism

\section{Changes in growth hormone and prolactin levels}

Thirteen studies [5-8, 10-11, 15-21], involving a total of 333 patients, reported the occurrence of low growth hormone levels in $\mathrm{LYH}$ patients. Sixteen studies [5-12, 14-21], involving a total of 360 patients, reported the occurrence of hyperprolactinemia in $\mathrm{LYH}$ patients. In both study series, heterogeneity analysis revealed that there was statistical heterogeneity; thus, the random effects model was used for combined analysis. The results showed that the incidence of growth hormone deficit in $\mathrm{LYH}$ patients was $0.22(95 \% \mathrm{Cl}$ : 0.13-0.32) and the incidence of hyperprolactinemia in LYH patients was 0.29 (95\% Cl: 0.19-0.39) (Figure 5). Three studies $[5,7,17]$ reported low prolactin levels in some patients with an incidence rate of 0.18 (95\% Cl: 0.08-0.28).

\section{Central diabetes insipidus}

All 17 studies [5-21], involving a total of 364 patients, reported the occurrence of central diabetes insipidus (CDI) in LYH patients. Heterogeneity analysis revealed that there was statistical heterogeneity among these 17 studies; thus, the random effects model was used for combined analysis. The results showed that the incidence of $\mathrm{CDI}$ in $\mathrm{LYH}$ patients was 0.45 (95\% Cl: 0.36-0.55) (Figure 6).

\section{Magnetic resonance imaging findings}

Ten different MRI characteristics of LYH were reported in 16 studies [5-10, 12-21], and each of these MRI characteristics was reported in between 2 and 13 of the 16 studies. The results showed that the commonest MRI characteristics of $\mathrm{LYH}$ were pituitary contrast enhancement (63\%), symmetric pituitary enlargement (60\%), thickening of the pituitary stalk (58\%), sella mass or suprasellar extension (58\%), loss of posterior pituitary hyperintensity (50\%), chiasmal compression (38\%), central pituitary necrosis (24\%), cystic appearance (17\%), cavernous sinus compression $(17 \%)$, and dural tail sign (9\%). The incidence of different MRI findings is shown in Table III.

\section{Treatment}

\section{Treatment with glucocorticoids and surgery}

All 17 studies [5-21] reported glucocorticoids and surgery in the treatment of $\mathrm{LYH}$ patients. Heterogeneity analysis revealed that there was statistical heterogeneity among these 17 studies; thus, the random effects model was used for combined analysis. The results showed that $36 \%(95 \% \mathrm{Cl}$ : 0.20-0.52) of LYH patients were treated with steroids, which was mainly glucocorticoid pulse therapy, with $34 \%$ (95\% Cl: $0.23-0.45)$ of LYH patients being treated surgically (Figure 7 ). 


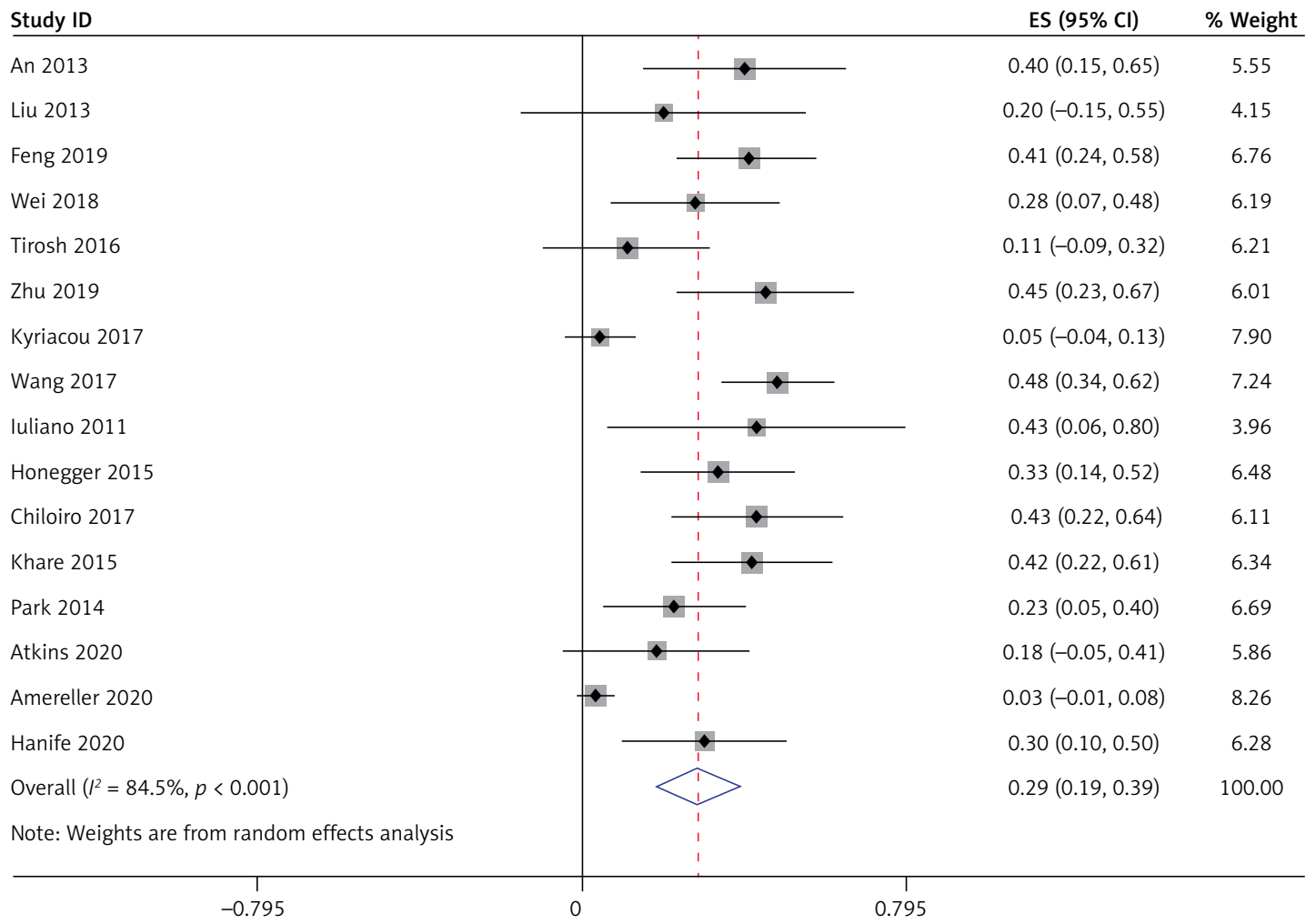

Figure 5. Incidence of hyperprolactinemia

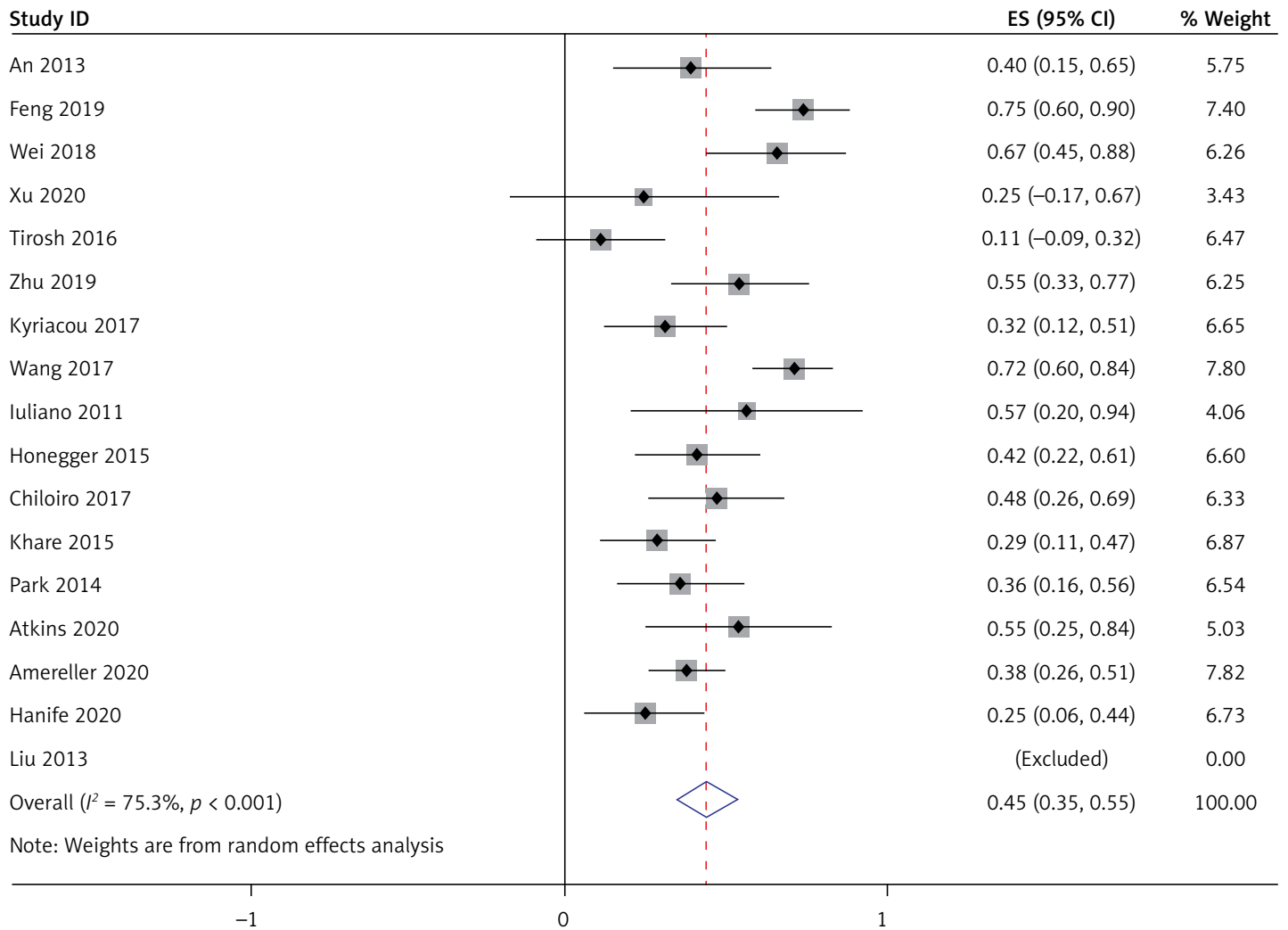

Figure 6. Incidence of central diabetes insipidus 


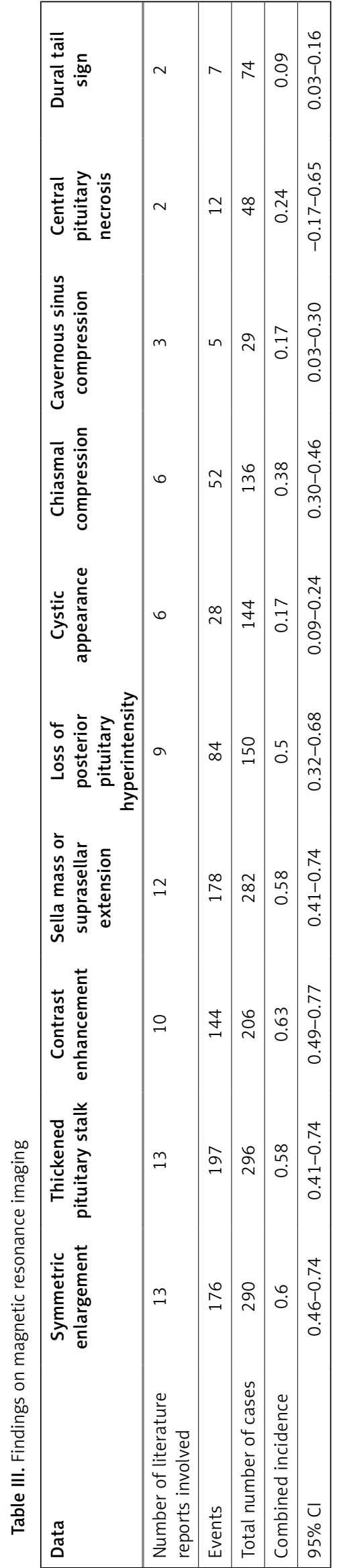

\section{Clinical follow-up observation or hormone} replacement therapy

Nine studies [5, 8, 9, 11, 15, 17-20], involving a total of 239 patients, reported LYH patients receiving clinical follow-up observation or hormone replacement therapy. Heterogeneity analysis revealed that there was statistical heterogeneity among the nine studies; thus, the random effects model was used for combined analysis. The results showed that $43 \%(95 \% \mathrm{Cl}: 0.22-0.64)$ of $\mathrm{LYH}$ patients received clinical follow-up observation or hormone replacement therapy.

\section{Discussion}

Hypophysitis is a rare autoimmune disease characterized by inflammation and cell infiltration of the pituitary gland. It can be divided into primary and secondary hypophysitis, with LYH being the most common type of primary hypophysitis. In the last ten years, an increasing number of clinical studies and case series have been published. The clinical manifestations of $\mathrm{LYH}$ are related to the severity of inflammation and the mass effect of the lesion, giving rise to four symptom categories: (1) symptoms resulting from sellar mass compression, such as headache, nausea, vomiting, and visual field impairment. Our analysis showed that the incidence of headache was $49 \%$ in $364 \mathrm{LYH}$ patients and the incidence of visual field loss and visual impairment was $26 \%$ in $350 \mathrm{LYH}$ patients, (2) symptoms of anterior pituitary hormone deficiency, where clinical presentation will vary depending on the hormone or hormones involved. Secondary hypoadrenalism can be manifest as weakness, fatigue, loss of appetite, nausea, vomiting, hypotension, and hypoglycemia. Secondary hypothyroidism can result in dry skin, cold intolerance, pallor, and reduced reaction times. Secondary hypogonadism can present with amenorrhea and loss of sexual desire. Our analysis showed that in $364 \mathrm{LYH}$ patients, the incidence of secondary hypogonadism, secondary hypoadrenalism, secondary hypothyroidism, and growth hormone deficit was $54 \%, 49 \%, 43 \%$, and $22 \%$, respectively, (3) posterior pituitary involvement leads to partial or total CDI, manifest as polyuria and polydipsia. In our analysis, CDI occurred in $45 \%$ of $364 \mathrm{LYH}$ patients, and (4) when the lesion involves the pituitary stalk, CDI, amenorrhea, galactorrhea, and other symptoms appear. In our analysis, hyperprolactinemia and low prolactin levels occurred in $45 \%$ and $18 \%$ of patients, respectively. LYH is common in women in late pregnancy and the early postpartum period: a case series reported prevalence of female gender among affected patients of between $52 \%$ and $71 \%$ [22]. An association with pregnancy was found in 5\% [23]. Our analy- 


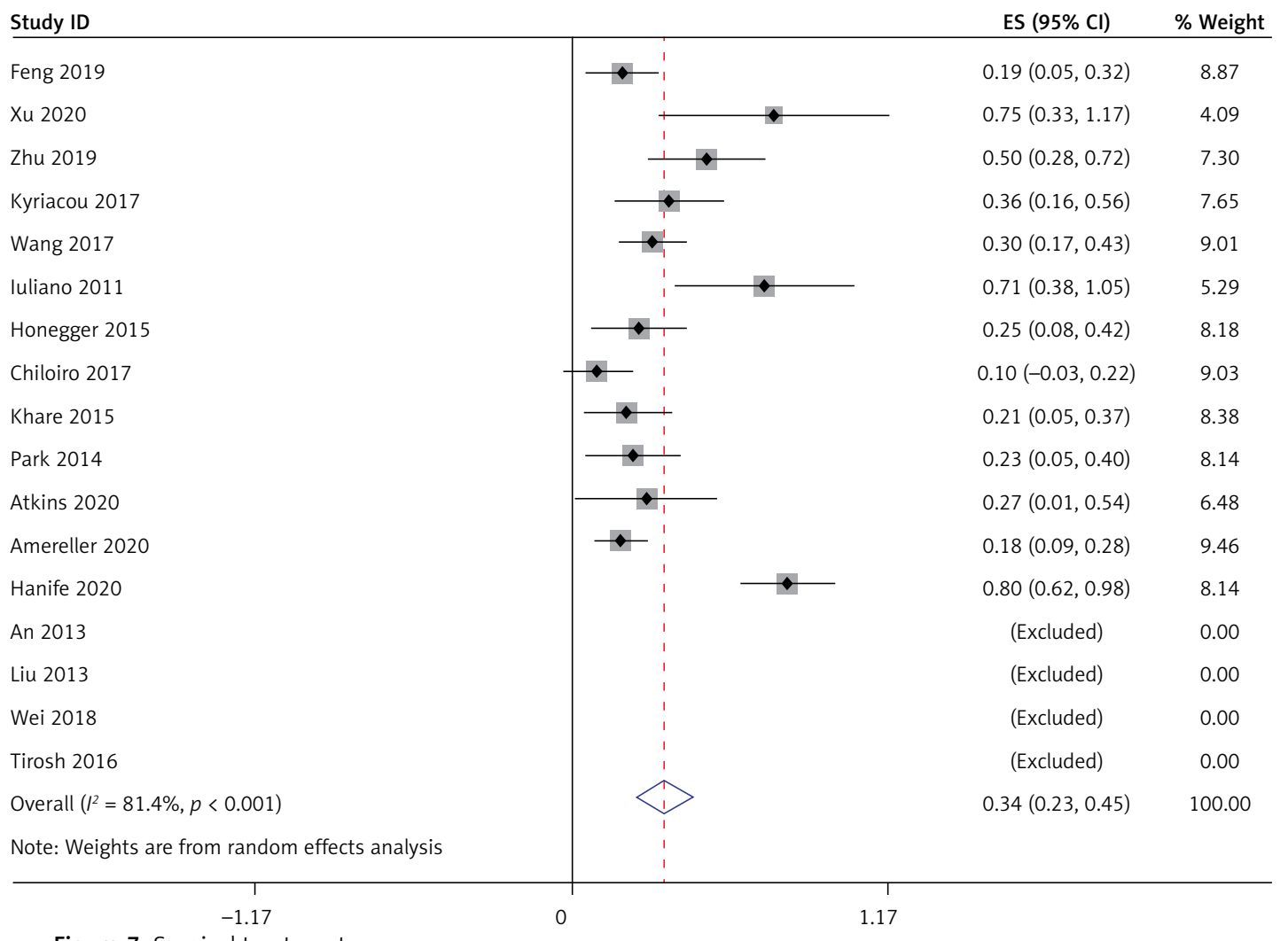

Figure 7. Surgical treatment

sis showed that the percentage of women in LYH patients was $78 \%$, and LYH was associated with pregnancy in $15 \%$ of female patients. However, in recent years, cases have been reported in men and women of all ages. LYH is also associated with systemic autoimmune diseases: previous studies have shown an association in approximately 30$42 \%$ of LYH cases [24]. In our analysis, the figure was $24 \%$ and included Hashimoto's thyroiditis, rheumatoid arthritis, Sjogren disease, systemic lupus erythematosus, Graves' disease, psoriasis, and Guillain-Barre syndrome.

Magnetic resonance imaging is the imaging modality of first choice for saddle area lesions as it can clearly identify the morphology, size, and signal characteristics of the pituitary via coronal and sagittal views. MRI can accurately evaluate the growth pattern, invasion range, and the relationship between the lesion and the surrounding tissue. The signal changes of the neurohypophysis, the shape and position of the pituitary stalk, and the bone substance changes can also be clearly displayed [25]. LYH is often misdiagnosed as pituitary hyperplasia, pituitary tumor, or other diseases. The main manifestation of pituitary hyperplasia is diffuse enlargement of the pituitary gland: this returns the same uniform parenchymal signal and uniform enhancement as the normal pituitary gland on MRI. Compared with $\mathrm{LYH}$, pituitary tumors have a distinct lesion boundary and demonstrate delayed mild enhancement while the surrounding normal pituitary tissue shows obvious enhancement. The MRI manifestations of LYH in our analysis were pituitary contrast enhancement (63\%), symmetrical pituitary enlargement (60\%), thickening of the pituitary stalk (58\%), sella mass or suprasellar extension (58\%), loss of posterior pituitary hyperintensity (50\%), chiasmal compression (38\%), central pituitary necrosis (24\%), cystic appearance (17\%), cavernous sinus compression (17\%), and dural tail sign (9\%). Some authors regard the dural tail sign with retrodural involvement and enhancement as one of the signs of LYH [26].

Lymphocytic hypophysitis usually presents as a progressive process. Initially, inflammation and edema lead to enlargement of the pituitary gland and the patient presents with symptoms of mass effect. When the pituitary cells are destroyed and the glandular parenchyma is fibrotic, the pituitary atrophies, resulting in hypopituitarism. At present, the main treatment methods for $\mathrm{LYH}$ include steroid therapy, surgical therapy, immunosuppressive medical therapy, targeted therapy, and observation with hormone replacement therapy alone. Glucocorticoids can reduce inflammation, thereby reducing any space-occupying effects, and are available for diagnostic treatment in approximately $30 \%$ of patients [27], although long-term effects are not clear. There is no unified recommended 
scheme for the treatment of LYH. For patients with severe or progressive exacerbation of acute or clinical symptoms, immunosuppressive agents based on glucocorticoids and surgery are the most commonly used active treatment for reducing masses. The commonly used regimen is highdose prednisone or methylprednisolone shock therapy, followed by a dose decreasing stage of weeks and months. Wei's study showed that [10] the effective rate of glucocorticoid treatment was $54.5 \%$. The main problems relating to steroid therapy are relapse after treatment and systemic complications caused by high dose or long-term use of glucocorticoids. Surgery can confirm the pathological diagnosis and can also remove pathological tissue to achieve decompression. Indications for surgical treatment include no response following steroid therapy, recurrence after remission, larger lesions, and compression symptoms such as visual loss and visual field defects. The main problems of surgical treatment are as follows [28]: (1) hypopituitarism will occur following surgery requiring lifelong hormone replacement therapy, and (2) the risk of surgical complications, such as cerebrospinal fluid rhinorrhea, infection, etc. Immunosuppression and targeted therapy are generally used for patients who have failed to respond to steroid therapy and surgery. Treatment with cyclophosphamide, methotrexate and rituximab has been reported [29]. However, due to the small number of cases, the specific drug regimen needs further study. Hormone replacement therapy is recommended when hormone deficiency or hypopituitarism occurs. Hormone replacement therapy is to supplement the corresponding target hormone of physiological dose, mainly glucocorticoid, thyroid hormone, sex hormone and desmopressin. Patients with pituitary hormone deficiency should receive long-term hormone replacement therapy, with more than $70 \%$ of patients requiring long-term hormonal substitution [5]. During this treatment, hormone levels should be monitored regularly, and drug doses should be adjusted as required. Patients with CDI should be treated with desmopressin or vasopressin supplementation. In addition, bromocriptine should be used in patients with hyperprolactinemia. It has been reported that spontaneous remission can occur in partial LYH cases: the pituitary mass can shrink spontaneously, anterior pituitary function can gradually recover in approximately $30 \%$ of patients, and, in a small number of cases, CDI can improve [30]. Therefore, for patients presenting with mild symptoms, watchful waiting may be a therapeutic option as recovery of hormonal function and improvements in imaging parameters may occur during the course of the disease [31]. Considering the limitations of steroid pulse therapy and surgical treatment, some scholars advocate that observation and follow-up may be applied to a wider population. Unless symptoms are severe or disease progression occurs, observation and follow-up is the preferred treatment for LYH patients [4]. For patients with simple pituitary stalk thickening and CDI, it is found that the clinical outcomes of the observation group and the immunosuppressant treatment group are similar through follow-up. Therefore, such patients should be observed and closely followed up [10]. If $M R I$ findings, endocrine function, or neurological symptoms indicate progression during follow-up, further intervention will be necessary. Our analysis showed that of $239 \mathrm{LYH}$ patients, 36\% were treated with steroids (mainly glucocorticoid pulse therapy), $34 \%$ were treated surgically, and $43 \%$ received clinical follow-up with observation or hormone replacement therapy.

In conclusions, lymphocytic hypophysitis is a relatively rare organ specific autoimmune disease characterized by extensive lymphocyte in filtration. It is easy to misdiagnose, and relapse after steroid treatment is common, as is pituitary dysfunction following surgery. The clinical manifestations include symptoms of mass effect and endocrine dysfunction. The characteristic MRI findings are an important basis for the clinical diagnosis of $\mathrm{LYH}$. With continuous progress in diagnostic technology and the accumulation of clinical experience in diagnosis and treatment, the prognosis of LYH and quality of life for $\mathrm{LYH}$ patients will continue to improve. Because the research time and research background of the included literature are different, the normal reference range of hormones in different laboratories is different, the quality of some included literature is not high, and there is certain heterogeneity between different studies, the results of this meta-analysis should only be used as a reference for clinical work, and more large-sample, multicenter, high-quality and well-designed randomized controlled studies are required.

\section{Acknowledgments}

Jia-Sheng Ju and Tao Cui contributed equally to this study.

\section{Conflict of interest}

The authors declare no conflict of interest

\section{References}

1. Chiloiro S, Capoluongo ED, Tartaglione T, et al. The changing clinical spectrum of hypophysitis. Trends Endocrinol Metab 2019; 30: 590-602.

2. De Bellis A, Ruocco G, Battaglia M, et al. Immunological and clinical aspects of lymphocytic hypophysitis. Clin Sci (Lond) 2008; 114: 413-21. 
3. Gutenberg A, Larsen J, Lupi I, Rohde V, Caturegli P. A radiologic score to distinguish autoimmune hypophysitis from nonsecreting pituitary adenoma preoperatively. AJNR Am J Neuroradiol 2009; 30: 1766-72.

4. Honegger J, Buchfelder M, Schlaffer S, et al. Treatment of primary hypophysitis in Germany. J Clin Endocrinol Metab 2015; 100: 3460-9.

5. Tirosh A, Hirsch D, Robenshtok E, et al. Variations in clinical and imaging dindings by time of diagnosis in females with hypopituitarism attributed to lymphocytic hypophysitis. Endocr Pract 2016; 22: 447-53.

6. Zhu Q, Qian K, Jia G, et al. Clinical features, magnetic resonance imaging, and treatment experience of $20 \mathrm{pa}$ tients with lymphocytic hypophysitis in a single center World Neurosurg 2019; 127: e22-9.

7. Kyriacou A, Gnanalingham K, Kearney T. Lymphocytic hypophysitis: modern day management with limited role for surgery. Pituitary 2017; 20: 241-50.

8. Wang S, Wang L, Yao Y, et al. Primary lymphocytic hypophysitis: clinical characteristics and treatment of 50 cases in a single centre in China over 18 years. Clin Endocrinol 2017; 87: 177-84.

9. Iuliano SL, Laws ER. The diagnosis and management of lymphocytic hypophysitis. Expert Rev Endocrinol Metab 2011; 6: 777-8.

10. Qian W, Guoqing Y, Yijun L, et al. Clinical features and prognosis of 18 cases of primary lymphocytic hypophysitis. Natl Med J China 2018; 98: 102-8.

11. Jie F, Saichao Y, Dongming Y. Clinical analysis of 32 cases of lymphocytic hypophysis. The Journal of Practical Medicine 2019; 35: 2065-9.

12. Xianglian A, Zhihong G, Mingcai Q. Early detection and management of lymphocytic hypophysis. Chin J Prey Contr Chron Dis 2013; 21: 471-4.

13. Bing X, Xiangzhong $\mathrm{H}$, Fan Z. Diagnosis and treatment of lymphocytic hypophysitis (report of four cases). Chin J Stereotact Funct Neurosurg 2020; 33:104-5.

14. Yanxia L, Qing Y, Xialian L, et al. Clinical analysis of $5 \mathrm{ca}-$ ses of lymphocytic hypophysis. Chin J Gen Pract 2013; 12: 752-3.

15. Honegger J, Schlaffer S, Menzel C, et al. Diagnosis of primary hypophysitis in Germany. J Clin Endocrinol Metab 2015, 100: 3841-9.

16. Chiloiro S, Tartaglione T, Angelini F, et al. An overview of diagnosis of primary autoimmune hypophysitis in a prospective single-center experience. Neuroendocrinology 2017; 104: 280-90.

17. Khare S, Jagtap VS, Budya SR, et al. Primary (autoimmune) hypophysitis: a single centre experience. Pituitary 2015; 18: 16-22.

18. Park SM, Bae JC, Joung JY, et al. Clinical characteristics, management, and outcome of 22 cases of primary hypophysitis. Endocrinol Metab 2014; 29: 470-8.

19. Atkins P, Ur E. Primary and ipilimumab-induced hypophysitis: a single-center case series. Endocr Res 2020; 54 246-53.

20. Amereller F, Küppers AM, Schilbach K, Schopohl J, Störmann S. Clinical characteristics of primary hypophysitis - a single-centre series of 60 cases. Exp Clin Endocrinol Diabetes 2020; 129: 234-40.

21. Oguz SH, Soylemezoglu F, Sendur SN, et al. Clinical characteristics, management, and treatment outcomes of primary hypophysitis: a monocentric cohort. Horm Metab Res 2020; 52: 220-7.

22. Imber BS, Lee HS, Kunwar S, Blevins LS, Aghi MK. Hypophysitis: a single-center case series. Pituitary 2015; 18: 630-41.
23. Angelousi A, Cohen C, Sosa S, et al. Clinical, endocrine and imaging characteristics of patients with primary hypophysitis. Horm Metab Res 2018; 50: 296-302.

24. Beressi N, Beressi JP, Cohen R, Modigliani E. Lymphocytic hypophysitis. A review of 145 cases. Ann Med Interne (Paris) 1999; 150: 327-41.

25. Ralot TK, Aggarwal J, Haniadka R, Gehlot K, Dongre N, Patil S. Lymphocytic hypophysitis mimicking pituitary macroadenoma. J Assoc Physicians India 2017; 65: 85-6.

26. Pekic S, Bogosav V, Peker S, et al. Lymphocytic hypophysitis? Successfully treated with stereotactic radiosurgery: case report and review of the literature. J Neurol Surg A Cent Eur Neurosurg 2018; 79: 77-85.

27. Korkmaz OP, Sahin S, Ozkaya HM, et al. Primary hypophysitis: experience of a single tertiary center. Exp Clin Endocrinol Diabetes 2021; 129: 14-21.

28. Karimnejad K, Sweeney J, Mantisdel JL. Cerebral vasospasm following transsphenoidal hypophysectomy in the treatment of lymphocytic hypophysitis. J Craniofac Surg 2016; 27: 988-91.

29. Xu C, Ricciuti A, Caturegli P, Keene CD, Kargi AY. Autoimmune lymphocytic hypophysitis in association with autoimmune eye disease and sequential treatment with infliximab and rituximab. Pituitary 2015; 18: 441-7.

30. Gilis-Januszewska A, Kluczyński Ł, Rogoziński D, Hubalewska-Dydejczyk A. Radiological and hormonal improvements in a 22-year-old patient with lymphocytic hypophysitis - the watchful waiting approach. Endokrynol Pol 2020; 71: 104-5.

31. Kluczyński Ł, Gilis-Januszewska A, Rogoziński D, Pantofliński J, Hubalewska-Dydejczyk A. Hypophysitis - new insights into diagnosis and treatment. Endokrynol Pol 2019; 70: 260-9. 